\title{
Chronique de la Grande Guerre de Maurice Barrès : une épreuve de l'anonyme
}

Denis Pernot

\section{(e) OpenEdition \\ 1 Journals}

Édition électronique

URL : http://journals.openedition.org/edl/1066

DOI : $10.4000 /$ edl. 1066

ISSN : 2296-5084

Éditeur

Université de Lausanne

\section{Édition imprimée}

Date de publication : 15 septembre 2017

Pagination : 145-160

ISBN : 978-2-940331-65-9

ISSN : 0014-2026

\section{Référence électronique}

Denis Pernot, "Chronique de la Grande Guerre de Maurice Barrès : une épreuve de l'anonyme », Études de lettres [En ligne], 2 | 2017, mis en ligne le 15 septembre 2019, consulté le 15 décembre 2020. URL http://journals.openedition.org/edl/1066 ; DOI : https://doi.org/10.4000/edl.1066 


\section{CHRONIQUE DE LA GRANDE GUERRE DE MAURICE BARRÈS : UNE ÉPREUVE DE L'ANONYME}

Revenant à cette part, désormais très oubliée, de son œuvre, notre réflexion envisage les chroniques de guerre que Barrès a publiées dans L'Écho de Paris sur le fondement d'une difficulté qu'il évoque à plusieurs reprises: comment célébrer des mérites anonymes? Comment inscrire dans la mémoire nationale française des héros qui ne peuvent être nommés? Elle montre que, prenant les aspects d'une épreuve de l'anonyme en action et en travail, ses chroniques de guerre conduisent Barrès à remettre en question, si ce n'est à refuser, l'autorité qui était associée à son nom: la célébration des mérites anonymes passe par un travail d'écriture amenant l'écrivain lorrain à jeter le voile de l'anonyme sur sa propre prise de parole.

Tels qu'il les rassemble dans deux éditions livresques successives, L'Âme française et la guerre (12 tomes, 1915-20) puis Chronique de la grande guerre (14 tomes, 1920-24) ${ }^{1}$, auxquelles s'ajoutent les pages réunies dans Les Diverses Familles spirituelles de la France (1917), les centaines d'articles que Barrès donne à L'Écho de Paris pendant toute la durée du conflit mondial forment une exceptionnelle œuvre de presse. Tirant profit de ses expériences de publiciste ${ }^{2}$, Barrès y fait usage de tous les moyens

I. Les citations des chroniques de Barrès données dans les pages qui suivent sont référées par leur titre et leur date de rédaction selon les indications fournies dans l'édition de Chronique de la Grande Guerre (Plon, 1920-1924) abrégé en CGG, avec indication de tomaison en chiffres romains et de pagination en chiffres arabes. Pour une présentation générale des chroniques de guerre de Barrès, voir V. Rambaud, «Maurice Barrès ».

2. Sur ce point, voir J. O. McShine, Barrès journaliste et V. Rambaud, «Les débuts de la collaboration de Maurice Barrès à L'Écho de Paris (pour une relecture du célèbre 
journalistiques de sorte que s'y mêlent des enquêtes, des interviews, des revues bibliographiques, des reportages et des nécrologies. Lues en continuité, ses chroniques prennent ainsi, à elles seules, les aspects dispersés et discontinus d'un quotidien auquel le travail d'une double préoccupation donne cohérence. Comme le montrent les pages où il dramatise les enjeux du conflit et en raconte de hautes scènes, Barrès y travaille à faire accepter à ses lecteurs, de l'arrière ou de l'avant, les sacrifices que l'effort de guerre exige d'eux. Comme le signale la nécessité qu'il a ressentie de les arracher aux colonnes de L'Écho de Paris, elles sont aussi tournées vers l'avenir d'une paix à construire. Barrès y mène donc conjointement deux projets, ce qui conduit à un éclatement de sa posture auctoriale: celui d'un archiviste soucieux de produire des documents aux historiens de l'avenir, celui d'un aède qui, s'appuyant sur la même matière, souhaite agir en "mainteneur de fraternité » ${ }^{3}$. Aussi rapproche-t-il son travail et ses intentions de ceux de l'épopée homérique:

La guerre anonyme fait place au siège de Troie. Et la méthode qu'avait le vieil Homère de dénombrer successivement les batailles et les héros, et de parcourir tous les lieux de la bataille en montrant au-dessus des hommes les idées-drapeaux et les dieux qui y assistent, donnerait seule une image de ce tourbillon, satisferait seule la vénération des familles françaises ${ }^{4}$.

Il constate toutefois qu'il lui est impossible de s'approprier la «méthode du vieil Homère» qu'il envisage comme un travail de dénombrement et de nomination. Cette impossibilité tient au contrôle que la censure exerce sur l'information ainsi qu'à sa situation discursive, celle d'un homme de l'arrière qui ne connaît les conditions de la bataille que par la visite de secteurs du front que les autorités veulent bien montrer et le cours des opérations militaires que par les communiqués qui en sont donnés. Tenant leurs rédacteurs pour des «abréviateurs [...] incapables d'aucun écart d'imagination ", Barrès situe sa parole vis-à-vis de celle des autorités qui les dictent:

article sur Les mains jointes)».

3. M. Barrès, "Des mainteneurs de fraternité», 10 septembre 1914, CGG, I, p. 197202.

4. Id., "Le voyage de Champagne (deuxième article)", 7 novembre 1915, CGG, VI, p. $251 s q$. 
Êtes-vous sûrs qu'on laisse jaillir devant nous toute la flamme de la tragédie nationale? [...] les communiqués [...] possèdent une grande qualité, la première de toutes: ils sont vrais [...]. Mais, pour être exact, faut-il être aride, sec et nu? ${ }^{5}$

Dans ces conditions, il est conduit à se demander comment célébrer des héros sans pouvoir les nommer et comment en nommer sans porter atteinte à la valeur de leur leçon.

\section{Anonymat et nomination}

Alors qu'il ne cesse de se confronter à des phénomènes qui relèvent de l'anonyme, Barrès fait un usage parcimonieux du terme qu'il emploie le plus souvent sous la forme d'un adjectif venant qualifier un substantif pluriel ou à valeur collective auquel il attache une valeur méliorative. Il évoque ainsi les membres de l'état-major qui ont préparé la France au conflit comme des "serviteurs anonymes" ${ }^{6}$, les combattants du front comme des "sauveurs anonymes ${ }^{7}$ ou comme une "masse presque anonyme [de] braves gens" ${ }^{8}$. S'il est conçu comme une force qui conduit la France à la victoire, l'anonyme est également perçu comme un risque prenant la forme, dans la durée de la guerre, d'une menace de démoralisation des combattants ou de leurs proches et de démobilisation des énergies, mais aussi, pour le futur, d'une menace d'effacement de l'événement qui rendrait inutiles les sacrifices consentis par la «masse presque anonyme des braves gens". Aussi, posant que "tous [les combattants] se veulent anonymes" ${ }^{9}$ et admettant que "les héros acceptent l'anonymat ${ }^{10}$, Barrès doit-il prendre, au nom de l'avenir, le risque de lever l'anonymat de ceux qui luttent pour offrir des noms à la mémoire nationale sans pour autant prendre, au présent, celui d'enrayer la dynamique des forces qu'il fédère. Plusieurs chroniques font montre

5. M. Barrès, «N'abusons pas de la teinte kaki », 6 novembre 1914, CGG, II, p. 108.

6. Id., "Cet admirable État-Major», 19 août 1914, CGG, I, p. 135.

7. Id., "Pour ceux qui se battent», 18 septembre 1914, CGG, I, p. 233.

8. Id., «La fête du poilu», 30 juin 1915, CGG, V, p. 149.

9. Id., «La gratitude de Paris», 8 septembre 1914, CGG, I, p. 191.

Io. Id., «En sortant de Notre-Dame. Nouveaux documents sur la vie et la mort de Driant», 29 juin 1916, CGG, VIII, p. 262. 
de ses hésitations face à cette difficulté. Il lui arrive au cours des premiers mois du conflit de regretter de ne pouvoir nommer ceux qu'il évoque et de réclamer des noms aux autorités:

[...] nous voudrions connaître nommément ceux vers qui se tourne notre gratitude ardente. Des noms de chefs, des numéros de régiments! Le gouvernement reconnaît que l'on a besoin de saluer ces héros. Pourquoi nous interdit-il de les connaître, de distinguer leurs figures et de les nommer? [...] nous voulons connaître nos héros ${ }^{11}$.

"Un sergent de qui je voudrais bien savoir le nom» fait ainsi, peu après qu'il a ainsi été évoqué, l'objet d'un aperçu biographique qui le nomme et l'héroïse:

Ce héros s'appelle Henri Gallard, il est de son métier facteur des Postes à Angers, et, pour la guerre, il est sergent réserviste au $117^{\mathrm{e}}$ de ligne ${ }^{12}$.

Loin de se plaindre toujours des contraintes qu' impose la censure, Barrès en tire parfois profit. Racontant une visite qui le conduit dans les «tranchées de première ligne", il évoque les soldats qu'il y rencontre comme des êtres de "vaillance» et de "misère qu'ils nient", de sorte qu'il se montre, pour finir, moins en présence de combattants qu'en relation avec des symboles que leur anonymat lui permet d'identifier:

[...] ce vaste désert est rempli des images invisibles de la famille, de la Patrie, du Devoir et de l'Honneur. Je suis au milieu des saints de la France $^{13}$.

Comme le montrent ces exemples, Barrès se montre aussi attentif à la force fédératrice de l'anonyme qu'au pouvoir, excitateur de mémoire, de la nomination. Désireux et inquiet de nommer, il est conduit tantôt à jeter le voile de l'anonymat sur les hommes qu'il évoque, tantôt à le lever

II. M. Barrès, "On demande des noms", 14 mai 1915, CGG, IV, p. 282.

I2. Id., "Une médaille de bronze pour les braves», 26 novembre 1914, CGG, II, p. 184; «Le vrai Noël pour l'armée», 20 décembre 1914, CGG, II, p. 297. De manière similaire, Barrès nomme occasionnellement dans L'âme française et la guerre puis dans CGG des personnages dont l'identité a d'abord été tue dans L'Écho de Paris.

I3. M. Barrès, "Au milieu des saints de la France», 22 novembre 1914, CGG, II, p. 167. 
pour nourrir la mémoire de ses lecteurs d'un nom: "Je profite de mes moyens d'information pour dire: "accourez, ici est un grand mort" " ${ }^{14}$.

Plusieurs de ses interventions prennent ainsi les aspects de notices nécrologiques. À celles-ci, il donne des titres où il nomme le disparu qu'il salue: "Ernest Psichari mort au champ d'honneur»; "Charles Péguy mort au champ d'honneur "; "La Mort d'Émile Clermont "... Conscient de rendre hommage à des victimes qu'il connait, Barrès craint toutefois de s'exprimer en membre d'une corporation et de "sacrifier un peu, au profit des "intellectuels", la part de l'immense peuple des anonymes et des silencieux" ${ }^{15}$. Aussi fait-il en sorte d'élever ceux qu'il nomme au rang de héros représentatifs de diverses classes de soldats (les prêtres, les ligueurs, les territoriaux...), ce qu'indiquent les titres d'autres nécrologies: «La Mort d'Alfred Gabriel, socialiste patriote»; «Les Sacrifices de l'intelligence. - Pierre-Maurice Masson ». Procédant de cette manière, il parvient à célébrer des "dévouements obscurs" ${ }^{16}$ sans avoir à renoncer à donner des noms. Ses interventions peuvent en outre prendre les aspects d'un ossuaire d'encre quand il en vient à dresser des listes de noms de victimes de guerre:

[...] Gass, tué à Sapigneules, le 19 septembre; Glaize, mort au champ d'honneur; Georget, tué à Vauquois, le 3 mars; Gumery, tué le 28 septembre, à Saint-Thierry; Hillemacher, tué le 6 septembre à Blesmes $[\ldots]^{17}$.

À mesure qu'augmentent les pertes, il prend conscience qu'«il n'est au pouvoir d'aucun de nommer tous ceux qui doivent l'être " ${ }^{18}$ et que "nulle mémoire ne saurait retenir les noms innombrables [des] héros» ${ }^{19}$. Aussi réfléchit-il à d'autres moyens de mobiliser les énergies de ses

I4. M. Barrès, "Un caporal, docteur ès lettres médite dans la tranchée. La Mort d'Amédée Guiard", 26 mars 1916, CGG, VII, p. 295. Ce texte a auparavant servi de préface à A. Guiard, Le Carnet intime d'Amédée Guiard, Bloud et Gay, 1917.

I5. Id., "Le jeune chef et ses soldats», 25 février 1917, CGG, IX, p. 161.

I6. Id., "Les dévouements obscurs. François et Gabriel Laurentie, soldats de la territoriale», 28 mars 1916, CGG, VII, p. 305.

17. Id., "Chez les artistes morts pour la patrie», 26 juillet 1915, CGG, V, p. 274 sq.

I8. Id., "En sortant de Notre-Dame. Nouveaux documents sur la vie et la mort de Driant», 29 juin 1916, CGG, VIII, p. 262.

I9. Id., "Les Vosges transfigurées", 30 novembre 1915, CGG, VI, p. 348. 
concitoyens et à d'autres possibilités de maintenir leur mobilisation au lendemain de la victoire.

\section{Noms et voix}

Du fait de son statut d'homme multiple, Barrès se trouve en situation d'engager diverses campagnes de presse. Ce faisant, expression qui revient souvent sous sa plume, il fait en sorte de "donner voix" aux «sentiments ${ }^{20}$ ou aux «réclamations ${ }^{21}$ de diverses catégories de la population: aux alsaciens et aux lorrains qui demandent à être enrôlés; aux hommes d'une compagnie qui désirent une fanfare; aux responsables d'associations de soutien aux invalides de guerre et de diverses œuvres de secours qui attendent que soient levés des fonds... Dans le cadre des campagnes de presse qu'il conduit alors, Barrès s'arrête notamment à la question des distinctions, des citations militaires, qui individualisent les plus méritants des combattants, mais à celle aussi de décorations dont il voudrait qu'elles soient attribuées de façon à servir d'encouragement à l'heure où elles sont accordées et qu'elles deviennent, par la suite, le centre visible d'une mémoire nationale. Une série d'articles le montre ainsi militer pour la création de la Croix de guerre, puis, ayant obtenu gain de cause, pour celle d'un "ruban" permettant de distinguer les invalides de guerre des invalides du travail:

Je demande une médaille des invalides de la guerre pour qu'elle soit, sur la poitrine d'un soldat malheureux, le témoin, la preuve du sacrifice que la France lui a demandé aux heures difficiles et pour qu'elle soit, auprès de nous tous, un rappel muet et un «Souviens-toi» de la dette patriotique qui nous incombe à tous ${ }^{22}$.

Il consacre également un ensemble d'interventions à dénoncer un projet de loi exigeant que les corps des soldats non identifiés soient brûlés, l'«incinération obligatoire» lui apparaissant comme une manière de

20. M. Barrès, «L'élan suprême de notre armée», 23 octobre 1917, CGG, X, p. 243.

2I. Id., "La canaille du Bonnet rouge», 6 novembre 1917, CGG, X, p. 276.

22. $I d$., "Un ruban devrait désigner les invalides de la guerre», 28 mars 1915, CGG, IV, p. 74. 
démoraliser les combattants et leurs familles, mais aussi comme un vol de mémoire dont sont victimes les plus anonymes des soldats:

Déchirez cette loi qui atteindrait peu les officiers, car ils seront toujours aisément reconnus, mais qui priverait de sépulture un très grand nombre de simples soldats ${ }^{23}$.

De manière similaire, il voit la présence dispersée des tombes dans l'espace de la bataille de même que les inscriptions qu'elles portent comme l'occasion, toujours renouvelée et infiniment renouvelable, d'une mobilisation des cœurs et des esprits: "Une tombe est un enseignement. Il s'agit de donner au mort une voix pour qu'il nous instruise, nous émeuve et nous oblige» ${ }^{24}$. Dans le cadre d'une autre campagne de presse, il propose de transférer à leurs proches (parents, enfants, veuves) le droit de vote des victimes de guerre:

Ces morts [...] dont nous entendrons la voix jusqu'à la fin de nos jours, pouvons-nous accepter qu'ils se taisent désormais et qu'ils ne donnent aucun avis dans la reconstruction de la patrie qu'ils ont sauvée? [...] Je demande qu'ils puissent voter ${ }^{25}$.

Se trouvant dans l'impossibilité de nommer tous les morts qui méritent de l'être, il souhaite leur rendre la possibilité de s'exprimer en participant à la vie de la nation au-delà du sacrifice auquel ils ont consenti :

Les familles en deuil affirment avec nous que leur fils, leur époux, leur père, tombés pour la patrie, et dont elles entendent, chaque nuit, la voix, n'ont pas fini de pouvoir servir la France ${ }^{26}$.

Les voix de ces combattants anonymes, Barrès fait en sorte de les faire entendre à tous ses lecteurs. Il tire les informations qu'il leur livre de plusieurs sources, des témoignages qu'il recueille auprès des soldats, mais

23. M. Barrès, "Pour des braves gens qu'on empêche de pétitionner. Contre la Chambre qui veut brûler les morts», 20 juillet 1915, CGG, V, p. 246.

24. Id., "L'hommage aux morts et à leurs familles", $1^{\mathrm{er}}$ novembre 1917, CGG, X, p. 260.

25. Id., "Le suffrage des morts», $1^{\text {er }}$ février 1916, CGG, VII, p. 108.

26. Id., "De tels morts peuvent encore servir la France», 9 février 1916, CGG, VII, p. 135. Sur cette campagne qui nourrit plusieurs chroniques, voir M. Baumont, «Maurice Barrès et les morts de 1914-1918» et L. V. Smith, "Le corps et la survie d'une identité dans les écrits de guerre français». 
surtout des courriers qu'il reçoit et sollicite des combattants ou de leurs familles ${ }^{27}$. Il dispose ainsi d'une solution lui permettant de poursuivre sa double entreprise d'exaltation et de commémoration, solution qui consiste à leur céder la parole:

Toutes les lettres qui nous viennent de l'armée sont brûlantes. De chaque bataillon s'échappent les plus admirables histoires. La source est devant nous; il n'est que d'y plonger à plein seau pour en rapporter du sublime ${ }^{28}$.

Revenant sur le texte de la chronique où il dit regretter de ne pouvoir nommer les vainqueurs de la bataille de la Marne, il corrige son propos et met au jour la valeur des correspondances de guerre:

Je me rectifie. Nous ne connaissons pas les noms de nos défenseurs. C'est vrai. Mais que nous connaissons bien leur âme! Nous la voyons, nous l'entendons, nous la lisons. À chaque minute, une lettre, crayonnée hâtivement, nous apporte des nouvelles frémissantes, brûlantes, de nos fils, de nos frères, de nos maris, et nous livre tous les mouvements de ces cœurs de héros ${ }^{29}$.

Ce faisant, Barrès tire profit de l'impossibilité dans laquelle il se trouve de nommer les auteurs des lettres qu'il cite en indiquant que les voix qu'il fait entendre ne sont pas des voix individuelles, celles d'hommes engagés dans des conditions données, mais des voix d'anonymes qui sont la voix de l'unanime:

Laissez-moi verser au dossier qui fait notre confiance une pauvre petite lettre, crayonnée sur un lit d'hôpital, toute pareille d'âme et d'aspect à celles que lisent, avec tant d'orgueil et d'émotion, toutes les familles de France. Elle est d'un petit soldat, presque un enfant, un héros tout de même, et nous fait voir ce que sont les conscrits de $1914^{30}$.

Nombre de ses interventions se construisent ainsi autour de paroles qui ne sont pas siennes, qu'il se contente de présenter et qu'il fait rayonner

27. Sur ce point, voir V. Rambaud, «Comment composer une œuvre en dépouillant son courrier".

28. M. Barrès, «N'abusons pas de la teinte kaki», 6 novembre 1914, CGG, II, p. 109.

29. Id., "L'âme des soldats", 9 septembre 1914, CGG, I, p. 193.

30. Id., "Les conscrits de 1914 ", 15 septembre 1914, CGG, I, p. 220. 
en les multipliant: "Voici cette lettre [...]", "Je prends un autre cas, et vous mets sous les yeux une lettre qui se fait comprendre sans commentaire» ${ }^{31}$. Indiquant que "toutes [les] jeunes voix» qu'il fait porter "ne [font] que se répéter", il donne à croire qu'elles parlent d'une même voix. Aussi tient-il les différentes lettres qu'il cite pour les diverses "strophes" d'un "poème incomparable " ${ }^{32}$, l'ensemble des documents qu'il exploite pour les fragments d'un «sublime poème" qu'il situe dans la tradition de la chanson des geste, tradition où règne l'anonymat:

Dans la Chanson de Roland, seuls les chefs, Roland, Olivier, Turpin parlent durant la bataille; les autres se battent sans une parole; mais quand les silencieux, les anonymes sont morts, Dieu, sur le corps de chacun, fait "croistre un albespin". [...]. Et cette sainteté des mérites anonymes, nos chansons de gestes l'expriment à plusieurs reprises par de beaux symboles ${ }^{33}$.

Il donne ainsi aux lettres qu'il reproduit un statut littéraire qui les place dans la filiation des anonymes "poèmes idéalistes de notre moyen âge» ${ }^{34}$. Aussi, s'étant mis en situation de faire parler et d'honorer des héros anonymes, Barrès envisage-t-il la transmission de son entreprise journalistique sur le modèle de la veillée qu'il envisage comme une veillée quotidienne et nationale. Dans le cadre des formules de présentation des lettres ou des carnets qu'il rend publics, il emploie en effet, de préférence au verbe «lire», le verbe «écouter»: «Écoutez cette lettre $[. .$.$] » { }^{35}$; «Écoutez, voyez se dessiner [...]» ${ }^{36}$; «Écoutez, voyez ce que, d'un coup de dague sous le flot, nous ramenons ${ }^{37}$. Autour des lettres des héros anonymes qu'il cite, "tous les Français peuvent faire cercle» ${ }^{38}$ dans le moment de la bataille et dans les temps qui lui succèdent.

3I. M. Barrès, "Au service de nos soldats", 4 juin 1915, CGG, V, p. 38.

32. Id., "Le dialogue des jeunes guerriers et du chef», 18 juin 1915, CGG, V, p. 97.

33. Id., «Le jeune chef et ses soldats», 25 février 1917, CGG, IX, p. 165.

34. Id., "Les conscrits de 1914 », 15 septembre 1914, CGG, I, p. 222.

35. Id., «Les affinités franco-espagnoles», 7 janvier 1915, CGG, III, p. 40.

36. Id., "Le soldat sur les tombes", 15 janvier 1915, CGG, III, p. 79.

37. Id., "Solidité des Belges sous le flot allemand», 14 mars 1915, CGG, IV, p. 16.

38. Id., "L'âme des soldats", 9 septembre 1914, CGG, I, p. 194. 


\section{Extraire de l'anonymat et entrer dans l'anonymat}

Barrès comprend, dès les premières phases du conflit, que celui-ci ne peut se dire de la même manière que ceux qui l'ont précédé dans l'Histoire: "C'est fini de ces grandes popularités militaires [...] qui faisaient dire aux combattants, à toute la nation: "La Pucelle, Condé, Turenne, le Petit Tondu sont là!" " ${ }^{39}$. Aussi ne célèbre-t-il guère les hauts membres du commandement militaire. S'il lui arrive d'évoquer Joffre ou Foch, il leur préfère des figures moins connues qu'il extrait de l'anonymat. Il consacre ainsi plusieurs chroniques, au moment de sa mort, au colonel Driant, qu'il montre sous les traits d'un officier attentif à ses hommes, dont il indique qu'il a été son collègue au Parlement mais dont il se garde de rappeler, afin de ne pas nuire à l'Union sacrée, qu'il est le gendre du général Boulanger. Il exalte également la figure héroïque de Jacques Péricard, ce sous-officier qui, à Verdun, le 8 avril 1915, aurait conduit ses hommes à l'assaut en leur lançant un «Debout les morts» qu'il popularise par une chronique et deux préfaces. S’il invite ainsi ses lecteurs à faire cercle autour de héros qu'il crée, il les choisit moins souvent dans le monde du commandement militaire ou politique que dans celui d'hommes qui ont combattu par la plume avant de mourir par l'épée. Sont particulièrement significatives, à cet égard, les chroniques qu'il consacre à Péguy et à Psichari. À l'heure de leur disparition, aucun d'eux n'est familier aux lecteurs de L'Écho de Paris, de sorte que Barrès, qui associe le souvenir de leurs œuvres à celui de leur sacrifice, les extrait de l'anonymat et invente d'un même geste des héros et des écrivains. Il se tient toutefois en retrait de ce double geste d'invention héroïque et littéraire. Des chroniques qu'il consacre à Péguy, il ne prend en charge que la première, reproduite à l'envi dans la presse de l'époque ${ }^{40}$ et cède, pour les autres, la parole à un témoin des combats de Villeroy, Victor Boudon. Il n'assiste en outre ni aux commémorations orléanaises du premier anniversaire de la disparition de l'écrivain, ni à celles qui sont organisées le même jour sur le champ de la bataille. Barrès laisse ainsi à ceux qui font cercle autour de ses écrits le soin d'honorer le héros qu'il a créé, de l'inscrire dans un panthéon mobile aux côtés d'autres héros, restés anonymes, dont personne ne saurait énumérer les noms, ni arrêter une liste.

39. M. Barrès, "La gratitude de Paris», 8 septembre 1914, CGG, I, p. 189 sq. 40. Sur ce point, voir D. Pernot, "Charles Péguy mort au champ d'honneur"». 
De manière similaire, bien qu'il fasse connaître la disparition de Psichari, il abandonne à d'autres, Paul Bourget et Henri Massis, le soin de mener à son terme un travail d'hérö̈sation qu'il se contente d'engager. Il cherche ainsi moins à individualiser des héros qu'à leur faire rejoindre la communauté nationale telle qu'elle est définie par l'Union sacrée. Dans la mesure où, à ses yeux, les écrivains sont "les guides de la nation" tout autant que ses "secrétaires", dans la mesure où ils "écrivent sous la dictée de leurs concitoyens en même temps qu'ils propagent des manières de sentir " ${ }^{41}$, il les envisage comme des porte-parole de l'anonyme. Parce que leurs noms valent pour ceux des anonymes sous la dictée desquels ils ont écrit, il s'efforce de les inscrire dans les mémoires en obtenant de la Société des gens de lettres la création d'une médaille commémorative ou en présentant une anthologie qui réunit quelques-unes des pages qu'ils ont laissées :

[...] groupés autour d'eux en cercle, nous les regardons, nous les écoutons, figures douloureuses et charmantes, déjà devenues un peu mystérieuses pour nous-mêmes qui les avons familièrement connues. A demi rentrées dans l'ombre, ces âmes rayonnent doucement; épurées par la mort, elles se sont réunies avec l'idéal qu'elles poursuivaient au milieu de nous ${ }^{42}$.

Ce faisant, il montre «que la mort au champ de bataille fait d'un simple écrivain un maître» ${ }^{43}$ autour duquel il invite la postérité à faire cercle: "Cette lettre, on dirait une page extraite des Enfances de Tristan»" ${ }^{4}$. L'anonymat du héros combattant est consacré par celui de l'auteur de l'œuvre qui fait porter sa voix: le héros anonyme ne peut être dit que par un auteur qui accepte pour lui-même l'anonymat.

Aussi Barrès subordonne-t-il sa voix de secrétaire de la nation aux voix des héros anonymes sous la dictée desquels il écrit et se prive-t-il de toute légitimité:

4I. M. Barrès, "Une décision de l'Académie», 18 mars 1915, CGG, IV, p. 39. Sur l'idée que Barrès se fait désormais de la mission de l'homme de lettres, voir D. Pernot, "Barrès en guerre».

42. Id., "Pour une anthologie des écrivains tombés à l'ennemi", 15 février 1916, $C G G$, VII, p. 162 .

43. Id., "Que la mort au champ de bataille fait d'un simple écrivain un maître. La Foire de Lyon", 25 avril 1916, CGG, VIII, p. 51-58.

44. Id., "Les conscrits de 1914 », 15 septembre 1914, CGG, I, p. 222. 
[...] ces lettres me confondent; elles veulent me faire croire que je suis aussi un soldat [...] et je sais trop bien que, $s^{\prime}$ il est possible que je rende quelque service, il est faux que j’aie aucun mérite. Tout le mérite n'appartient qu'à ceux qui souffrent et dont je ne suis pas. [...] À côté d'eux, il faut se taire ${ }^{45}$.

Aux combattants qui demandent «quelques lignes" à l'écrivain qu'il reste à leurs yeux, il répond par "quelques citations» qu'il leur emprunte, par des "extraits de lettres" qu'il donne à "écouter" ${ }^{46}$. Il fait ainsi en sorte d'entrer autant que faire se peut dans l'anonymat, ce qui le conduit à indiquer qu'il ne s'exprime pas en son nom: "[...] il ne s'agit de personne, il s'agit de tout le monde» ${ }^{47}$. Aussi déclare-t-il volontiers s'exprimer en porte-parole et en secrétaire d'un héros qui n'a pas fait la guerre, ce qui lui permet de le nommer, et en retrait duquel il se place, Paul Déroulède. Chronique de la Grande Guerre s'ouvre en effet sur un événement discursivement fondateur, la mort du président de la Ligue des Patriotes à la tête de laquelle l'écrivain accède en juillet 1914. Evoquant le discours qu'il a prononcé le 4 mai 1913 à l'occasion de la fête de Jeanne d'Arc, Barrès subordonne sa parole à celle de Déroulède:

Tout en faisant le discours que je devais, je me disais: “C'est un peu fort que je parle quand Déroulède se tait." 48

Aussi est-ce la voix de Déroulède, autant que celle des combattants anonymes, plutôt que la sienne que Barrès veut faire rayonner quand il indique que la Ligue des Patriotes doit être le «secrétariat des soldats» ${ }^{49}$. Comme l'indique déjà son discours du 4 mai 1913, il prête toujours sa voix à Déroulède dans la dizaine de chroniques où il évoque la Pucelle, mais il l'y prête également à tous ceux qui ont célébré l'héroöne: à un historien comme Michelet, à un écrivain comme Péguy, à un antimilitariste rallié à l'effort de guerre comme Gustave Hervé ou à un radical comme Joseph Fabre qui a œuvré afin que soit instaurée une fête nationale de

45. M. Barrès, «Les héros s'entr'aident», 17 juillet 1915, CGG, V, p. 221.

46. Ibid., p. 221 sq.

47. M. Barrès, "Gallieni en septembre 1914», $1^{\text {er }}$ juin 1916, CGG, VIII, p. 207.

48. Id., "Le cahier rouge de Marcel Drouet mort au champ d'honneur», 13 mars 1915, L'âme française et la guerre, t. 4, p. 12. Ce passage n'est pas repris dans CGG.

49. Id., "Nous sommes le secrétariat des soldats (La Ligue des Patriotes)", 4 mars 1915, CGG, III, p. 319-323. 
Jeanne d'Arc. Mêlant ces voix, Barrès se fait le légataire universel de ce projet qui l'amène à voir et à faire voir en Jeanne une héroïne de l'Union sacrée. La Pucelle devient ainsi sous sa plume une figure lui permettant de désigner sans les nommer les anonymes qu'il ne peut individualiser. Portant leurs voix, elle est, pour finir, la voix de l'unanime:

[...] groupons-nous autour d'une vierge toute faite de vaillance et de bonté, de droiture et de sacrifice pour proclamer d'une voix unanime, Français de tous les partis, que le propre de la puissance est de délivrer et de protéger ${ }^{50}$.

Les écrits de guerre de Barrès sont, à plusieurs titres, une épreuve de l'anonyme qu'impose cette forme nouvelle de guerre qu'est la guerre industrielle de masse, qui se traduit par une "hécatombe " ${ }^{51}$ ou un "holocauste " ${ }^{52}$ des forces vives de la nation. Aussi ne s'agit-il pas seulement pour lui de nommer et de faire écouter des héros représentatifs, ce qu'il fait quand il évoque Déroulède comme un "apôtre " 53 ou quand il milite pour la création d'une fête nationale de Jeanne d'Arc, mais d'inscrire la guerre dans la mémoire nationale afin que chacun de ses lecteurs trouve la force morale de s'approprier la vertu de ses héros anonymes et de perpétuer leur vertu en renouvelant indéfiniment l'Union sacrée. Chronique de la Grande Guerre se présente ainsi comme une ouvre tout entière consacrée à garantir une "transfusion d'hérö̈sme " ${ }^{54}$ entre combattants de l'avant et gens de l'arrière, entre morts et vivants, mais aussi entre Barrès et les anonymes dont il porte la parole. Perçu comme un événement transfigurateur, le conflit y est pensé comme l'occasion d'une renaissance ou d'une résurrection permanente de la France à travers celle de la voix de ceux qui sont tombés au combat. À leur sacrifice, Barrès ressent l'impérieuse nécessité d'en adjoindre un autre, qui prend la forme d'un renoncement aux querelles du passé et aux positions acquises, d'un renoncement à être

50. M. Barrès, «La fête nationale de Jeanne d'Arc», 21 décembre 1914, CGG, II, p. 305. Sur ce point, voir D. Pernot, "Jeanne d'Arc dans les écrits de guerre de Maurice Barrès".

5I. Id., "La supériorité morale de la France», 8 mars 1915, CGG, III, p. 336.

52. Id., "Ceux qui se sacrifient ne veulent pas être dupes», 17 février 1915, CGG, III, p. 251.

53. Id., "Metz. Méditation au cimetière de Chambière», 29 novembre 1918, CGG, XIII, p. 262.

54. Id., "Le suffrage des morts», $1^{\text {er }}$ février 1916, CGG, VII, p. 109. 
l'écrivain et le polémiste qu'il a jusqu'alors été aux yeux de ses lecteurs. Interrogé sur l'avenir des lettres, il fixe ainsi à la littérature un pouvoir spirituel qu'il remet aussitôt aux combattants qui survivront au conflit, combattants dont les voix sauront porter et se faire entendre d'ellesmêmes:

Bientôt ils nous reviendront vainqueurs; ils prendront dans notre ordre, comme dans tous les ordres, la maîtrise et toutes les primautés: ce sera peu qu'ils nous racontent ce qu'ils sont en train de voir; ils nous ennobliront, en nous rendant accessibles les vertus dont ils sont, à cette heure, remplis 55 .

Ayant le sentiment d' "assist[er] à la dépossession des équipes d'hier " ${ }^{56}$, Barrès se confronte dans ses chroniques de guerre à une forme d'anonymat, qu'il choisit de faire peser sur son autorité, son œuvre et son nom, manière pour lui de se fondre dans «la masse presque anonyme des braves gens». Révélatrice est à cet égard une anecdote où il montre "deux soldats" anonymes le prendre pour un autre, le chansonnier Théodore Botrel, et où il indique n'attacher aucune importance à une erreur qui porte sur son identité mais non sur son rôle et sa mission, ce qui l'amène à demander à Botrel de lui "faire une place l'une de [ses] chansons» afin de faire entendre sa voix mieux que ses œuvres la font entendre ${ }^{57}$. À ceux qui sont morts au feu, Barrès offre ainsi un sacrifice symbolique, celui de sa voix qu'il cède aux anonymes dont il fait écouter les témoignages, celui de son autorité qu'il cède aux survivants. Ce sacrifice entraîne celui de son œuvre, ce que signalent par la suite les déclarations où il tient ses écrits de guerre pour l'unique part de sa production qui mérite de durer ${ }^{58}$, déclarations qui font qu'il passera longtemps aux yeux de la postérité, selon le surnom que lui donne Romain Rolland, pour un «rossignol du carnage».

Denis Pernot

Université Paris XIII

55. M. Barrès, "Au président de la Société des gens de lettres», 9 mars 1915, CGG, III, p. 345.

56. Id., «La France d'après la guerre», 7 mars 1915, CGG, III, p. 333.

57. Id., "Le chansonnier des armées», 21 octobre 1914, CGG, II, p. 35. Ce texte sert par la suite de préface aux Chants du bivouac (1915) de Botrel.

58. Sur ce point, voir F. Lefèvre, "Maurice Barrès", in Une heure avec... (première série), p. 27-36. 


\section{BIBLIOGRAPHIE}

\section{Textes}

BArrès, Maurice, L'Âme française et la guerre, t. 4, Paris, Emile-Paul, 1916.

-, Chronique de la grande guerre, 14 volumes, Paris, Plon, 1920-1924.

—, Les Diverses Familles spirituelles de la France, Paris, Emile-Paul, 1917.

Botrel, Théodore, Les Chants du bivouac, Paris, Payot, 1915.

Guiard, Amédée, Le Carnet intime d'Amédée Guiard, Paris, Bloud et Gay, «Pages actuelles», 1917.

LefÈvre, Frédéric, Une heure avec... (première série), Paris, Éditions de la NRF, 1924.

\section{Travaux}

Baumont, Michel, "Maurice Barrès et les morts de 1914-1918", L'Information historique, 1 (1969), p. 19-29.

McShine, J. Orville, Barrès journaliste, Port-au-Prince, Presses nationales d'Haïti, 1966.

Pernot, Denis, "Barrès en guerre», Travaux de littérature, 20 (2007), p. 461-473.

—, "Jeanne d'Arc dans les écrits de guerre de Maurice Barrès ", Le Porche (Orléans), 32 (mars 2010), p. 57-66.

—, " "Charles Péguy mort au champ d'honneur”. Discours nécrologique et mémoire immédiate (1914-1915)» in Pierre Allorant, Noëlline Castagnez (éds), Mémoires des guerres. Le Centre-Val-de-Loire de Jeanne d'Arc à Jean Zay, Presses universitaires de Rennes, 2015, p. 307-319.

Rambaud, Vital, «Les débuts de la collaboration de Maurice Barrès à L'Écho de Paris (pour une relecture du célèbre article sur Les mains 
jointes)", Nouveaux cahiers François Mauriac, 18 (2010), p. 185194.

—, "Comment composer une œuvre en dépouillant son courrier. L'Exemple de Chronique de la Grande Guerre de Maurice Barrès", www.fabula.org/colloques/document1750.php

—, "Maurice Barrès: une parole qui ne passe pas" in Romain Vignest, Jean-Nicolas Corvisier (dir.), La Grande Guerre des écrivains, Paris, Classiques Garnier, 2015, p. 71-84.

Smith, Leonard V., "Le corps et la survie d'une identité dans les écrits de guerre français ", Annales: Histoire, Sciences sociales, 1 (2000), p. 11-33. 